\title{
Mechanical Hybrid KERS Based on Toroidal Traction Drives: An Example of Smart Tribological Design to Improve Terrestrial Vehicle Performance
}

\author{
Francesco Bottiglione, ${ }^{1}$ Giuseppe Carbone, ${ }^{1}$ Leonardo De Novellis, ${ }^{2}$ \\ Luigi Mangialardi, ${ }^{1}$ and Giacomo Mantriota ${ }^{1}$ \\ ${ }^{1}$ Dipartimento di Meccanica, Matematica e Management, Politecnico di Bari, V.le Japigia 182, 70126 Bari, Italy \\ ${ }^{2}$ University of Surrey, Faculty of Engineering and Physical Sciences, Guildford, Surrey GU2 7XH, UK \\ Correspondence should be addressed to Giuseppe Carbone; carbone@poliba.it
}

Received 4 August 2012; Accepted 15 February 2013

Academic Editor: Michel Fillon

Copyright (C) 2013 Francesco Bottiglione et al. This is an open access article distributed under the Creative Commons Attribution License, which permits unrestricted use, distribution, and reproduction in any medium, provided the original work is properly cited.

\begin{abstract}
We analyse in terms of efficiency and traction capabilities a recently patented traction drive, referred to as the double roller fulltoroidal variator (DFTV). We compare its performance with the single roller full-toroidal variator (SFTV) and the single roller half-toroidal variator (SHTV). Modeling of these variators involves challenging tribological issues; the traction and efficiency performances depend on tribological phenomena occurring at the interface between rollers and disks, where the lubricant undergoes very severe elastohydrodynamic lubrication regimes. Interestingly, the DFTV shows an improvement of the mechanical efficiency over a wide range of transmission ratios and in particular at the unit speed ratio as in such conditions in which the DFTV allows for zero-spin, thus strongly enhancing its traction capabilities. The very high mechanical efficiency and traction performances of the DFTV are exploited to investigate the performance of a flywheel-based Kinetic Energy Recovery System (KERS), where the efficiency of the variator plays an important role in determining the overall energy recovery performance. The energy boost capabilities and the round-trip efficiency are calculated for the three different variators considered in this study. The results suggest that the energy recovery potential of the mechanical KERS can be improved with a proper choice of the variator.
\end{abstract}

\section{Introduction}

Recent developments in the automotive field are related to the design of drive-trains with the aim of improving the exploitation of the thermal engine, according to the requirements of reduction of fuel consumption and polluting emissions [1-3].

To achieve these targets, hybrid powertrains are being studied and developed. Among all possibilities, some research works claim that mechanical hybrids are more efficient and give the greatest advantages in terms of reduction of fuel consumption and polluting emissions. Several investigations have been made to estimate the effective benefits that such systems can give in mainstream cars and trucks at the present state-of-the-art. Computational results demonstrate that a fuel economy improvement up to $25 \%$ can be obtained in mainstream passenger cars and trucks, which can also be improved further with engine downsizing [4-7]. Continuously variable drives are the core of mechanical hybrids. Chain/belt continuously variable transmissions (CVTs) have been widely studied either theoretically either experimentally [8-10]; however, limitations of the maximum transmittable torque and of control possibilities made the toroidal traction variators a valid alternative for the development of CVT drivetrains [11]. A toroidal traction drive is made of input and output disks, which are coupled, respectively, with drive and driven shafts and shaped in such a way to realize a toroidal cavity. A power roller, rotating inside the toroidal cavity, is employed to transfer torque from the drive disk to the driven one, by means of shearing action of elastohydrodynamic oil film; furthermore, the tilting of the power roller allows shifting 


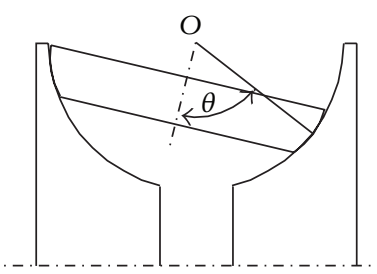

(a)

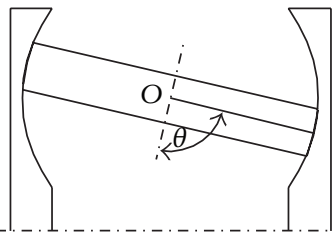

(b)

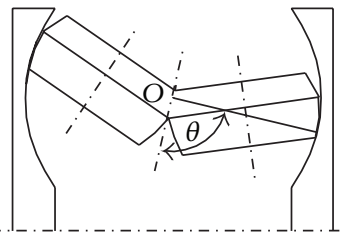

(c)

FIGURE 1: A schematic drawing of the three toroidal variators: the single roller half-toroidal variator (SHTV), (a); the single roller full-toroidal variator (SFTV), (b); the double roller full toroidal variator (c).

maneuvers. Referring to the toroidal variators which are actually on the market for automotive applications, the basic geometric distinction is on the position of the roller tilting center (point $O$ in Figure 1), which may coincide or not with the center of the toroidal cavity. In the first case one obtains the so called full-toroidal variator (see Figure 1(b)) in the second case the half-toroidal variator is obtained (see Figure 1(a)). In Figure 1(c), a new patented toroidal geometry [12], the so-called double roller full toroidal variator (or DFTV), is shown; two counter-rotating rollers are arranged inside the full-toroidal cavity, with the aim to reduce the spin losses at the roller-disk contact; also, the roller conical shape allows to balance the normal forces, thus making the employment of a thrust roller-bearing unnecessary. In this way, the main advantages of the two single-roller toroidal geometries (i.e., the SHTV and SFTV) can be combined leading to significant improvements of the overall transmission efficiency $[13,14]$. The ratio spread and the efficiency of the variable drives are key features for application to mechanical hybrid systems. A compromise between a large ratio spread and a good efficiency in both forward and reverse operation must be found to optimize the operating conditions of the KERS. It has been shown (see [15]) that shunted CVT architectures $([16,17])$ which enlarge the ratio spread of the variable drive, cannot improve the performance of the KERS as a consequence of a loss of efficiency, in particular in reverse operation (see also [18]). For these reasons, in this paper, we focus on the performance of standard toroidal traction drive geometries with speed ratio covering the range from 0.5 . to 2 . In particular, a fully flooded elastohydrodynamic-lubrication (EHL) model is presented to analyze, in terms of traction and efficiency performance, a recently patented toroidal traction drive variator (the DFTV) and compare it with more standard solutions as the single roller full-toroidal variator (SFTV) and the single roller half-toroidal variator (SHTV). The results are used to investigate the performance of a flywheel-based Kinetic Energy Recovery System (KERS) where the efficiency of the variator plays an important role in determining the overall energy recovery performance.

\section{Traction and Efficiency of Toroidal Drives}

In this section, we define the main geometric and kinematic characteristics of the toroidal variators. In Figure 2, $r_{0}$ is the first principal radius of the input and output disc, $r_{11}$ and $r_{33}$ are the second principal radii, respectively, of the input and output discs. In the case of the DFTV, each power roller has a conical part and a toroidal part; the rollers are in contact along the conical part, whilst the toroidal part is shaped as a typical half-toroidal roller and it is in contact with the input or output disc. We call $r_{2}$ the curvature radius of a section perpendicular to the roller axis, and $r_{22}$ is the second principal radius. For practical aspects, it is useful to define the conformity ratio $\mathrm{CR}=r_{22} / r_{0}$ and the aspect ratio $k=\mathrm{e} / r_{0}$, where $e$ is the eccentricity. An important control parameter is the tilting angle $\gamma$. We can express the dimensionless disks curvature radii formulation as a function of the cone angle $\theta$ and of the tilting angle $\gamma$ (see Figure 2) as $r_{11}=r_{1} / \cos (\theta+\gamma)$ and $r_{33}=r_{3} / \cos (\theta-\gamma)$, where $r_{1}=r_{0}(1+k-\cos (\theta+\gamma))$ and $r_{3}=r_{0}(1+k-\cos (\theta-\gamma))$. From geometric relations, we also obtain $r_{2}=\left(r_{0}+e\right) \sin (\alpha / 2)$. We also define the geometric speed ratio $s_{r_{\mathrm{ID}}}$ as the ratio of the radial coordinates $r_{1}$ and $r_{2}$; namely, $s_{r_{\mathrm{ID}}}=r_{1} / r_{3}$. Said $\omega_{1}$ the input angular velocity and $\omega_{3}$ the rotational speed of the output disc, we define the speed ratio $s_{r}$ as $s_{r}=\omega_{3} / \omega_{1}$. Percentage differences of the velocities of the sliding contact pairs are taken into account by defining input $C_{r_{\text {in }}}$ and output $C_{r_{\text {out }}}$ creep coefficients, respectively, that is, $C_{r_{\text {in }}}=\left(\omega_{1} r_{1}-\omega_{2} r_{2}\right) / \omega_{1} r_{1}$ and $C_{r_{\text {out }}}=\left(\omega_{2}^{\prime} r_{2}-\omega_{3} r_{3}\right) / \omega_{2}^{\prime} r_{2}$. The roller-roller slip coefficient is defined as $s_{c}=\omega_{2}^{\prime} / \omega_{2}$, where $\omega_{2}$ and $\omega_{2}^{\prime}$ are the angular velocities of the two counterrotating rollers. The transmission ratio can be expressed as

$$
s_{r}=\left(1-C_{r}\right) s_{c} s_{r_{\mathrm{ID}}}
$$

with $\left(1-C_{r}\right)=\left(1-C_{r_{\text {in }}}\right)\left(1-C_{r_{\text {out }}}\right)$. For each roller $i$ of the roller pair, we can define the point $\Theta_{i}$, which represents the point of intersection of the tangents to the toroidal cavity at the roller-disk contact points, and $\Omega_{i}$ is defined as the intersection point of the roller and disk rotation absolute axes. The angular velocities of the roller relative to the input and output disks, $\omega_{21}=\omega_{2}-\omega_{1}$ and $\omega_{23}=\omega_{2}^{\prime}-\omega_{3}$ have spin velocity components, $\omega_{21 \text { spin }}$ and $\omega_{23 \text { spin }}$, which can be directed inwards or outwards. By choosing a proper value of the cone incidence angle $\alpha$, it is possible to have zero spin velocities at $s_{r_{\mathrm{ID}}}=1$. The spin coefficients can be defined as

$$
\begin{gathered}
\sigma_{21}=\frac{\omega_{21 \text { spin }}}{\omega_{1}}=\sin (\theta+\gamma)-\left(1-C_{r_{\text {in }}}\right) \frac{1+k-\cos (\theta+\gamma)}{1+k}, \\
\sigma_{23}=\frac{\omega_{23 \text { spin }}}{\omega_{3}}=\sin (\theta-\gamma)-\frac{1}{\left(1-C_{r_{\text {out }}}\right)} \frac{1+k-\cos (\theta-\gamma)}{1+k} .
\end{gathered}
$$




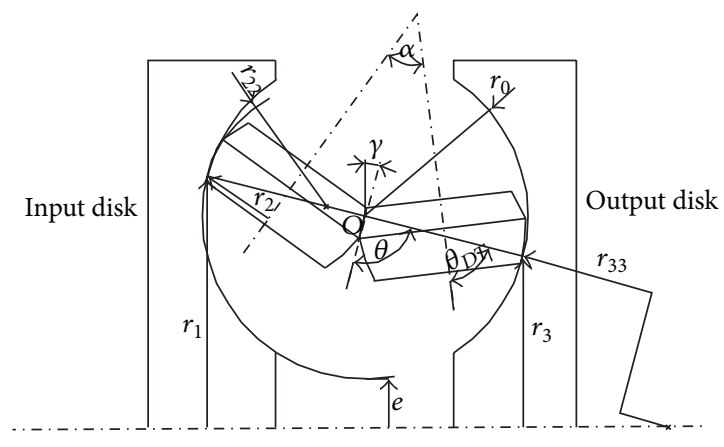

(a)

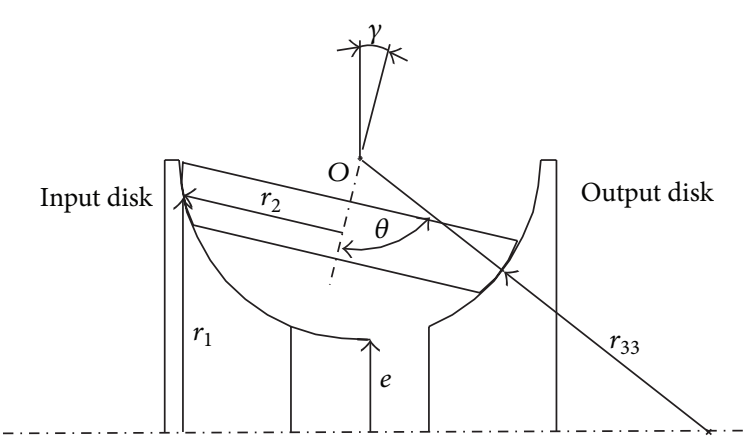

(b)

FIGURE 2: The geometry of the DFTV (a) and of the SHTV (b).

Following the same approach proposed in [13, 14], let us define the traction coefficient $\mu=F_{T} / F_{N}$ as the ratio between the tangential force at the roller-disk interface $F_{T}$ and the normal load $F_{N}$ and the spin momentum coefficient $\chi=$ $M_{S} / F_{N} r$, where $M_{S}$ is the spin momentum and $r$ the disk radial coordinate of the contact point. From the momentum equation applied to the counter-rotating rollers about each roller axis, the parameters $\mu$ and $\chi$ have been calculated according to $[13,14]$. Consider

$$
\begin{gathered}
\left(\mu_{\text {in }}-\mu_{c}\right)(1+k) \sin \left(\frac{\alpha}{2}\right)+\widetilde{r}_{1} \chi_{\text {in }} \sin \theta_{\mathrm{DT}}-t_{B}=0, \\
\left(\mu_{c}-\mu_{\text {out }}\right)(1+k) \sin \left(\frac{\alpha}{2}\right)+\widetilde{r}_{3} \chi_{\text {out }} \sin \theta_{\mathrm{DT}}-t_{B}=0,
\end{gathered}
$$

considering that the angle $\theta_{\mathrm{DT}}=(\pi-\alpha) / 2$ (see Figure 2$)$ and the radial coordinates in dimensionless form, namely, $\widetilde{r}_{1}=$ $r_{1} / r_{0}$ and $\widetilde{r}_{3}=r_{3} / r_{0}$. The term $t_{B}$ represents the dimensionless torque losses due to roller bearings on the variator roller axes, which have been evaluated according to the SKF technical documentation [19]. From the equilibrium equations of the discs, we can evaluate the effective torque coefficients at the input and output variator side as

$$
\begin{gathered}
t_{\text {in }}=\mu_{\text {in }}+\chi_{\text {in }} \sin (\theta+\gamma), \\
t_{\text {out }}=\mu_{\text {out }}-\chi_{\text {out }} \sin (\theta-\gamma),
\end{gathered}
$$

with $t_{\text {in }}=T_{\text {in }} /\left(m n F_{N} r_{1}\right)$ and $t_{\text {out }}=T_{\text {out }} /\left(m n F_{N} r_{3}\right)$ can be interpreted as effective torque coefficients at the input and output variator side. In the previous relations, we have considered the possibility to arrange $n$ set of rollers inside $m$ toroidal cavities. Moreover, we can express the thrust force acting in the disk axial direction $F_{D}$ as a function of the normal load $F_{N}$ and of the tilting angle $\gamma$ :

$$
\begin{aligned}
& \mathrm{F}_{D_{\text {in }}}=n F_{N} \sin (\theta+\gamma), \\
& F_{D_{\text {out }}}=n F_{N} \sin (\theta-\gamma) .
\end{aligned}
$$

The overall mechanical efficiency can be written as

$$
v=s_{c}\left(1-C_{r}\right) \frac{\mu_{\text {out }}-\chi_{\text {out }} \sin (\theta-\gamma)}{\mu_{\text {in }}+\chi_{\text {in }} \sin (\theta+\gamma)} .
$$

A fully flooded isothermal contact model $[13,14,20]$ has been utilized to calculate the shear stresses at the interface of the elements in contact, and thus, the traction and spin coefficients.

\section{The Contact Model}

We distinguish the contact conditions at the roller-disk interface, where the contact area is elliptical and at the roller-roller interface, where instead the contact area is rectangularly shaped. To study the contact between two curved profiles $a$ and $b$, we define an equivalent Young's modulus $E^{\prime}$, expressed as a function of the elasticity modulus $E$ and Poisson's ratio $v$ of each element in contact [20]

$$
E^{\prime}=2\left(\frac{1-v_{a}^{2}}{E_{a}}+\frac{1-v_{b}^{2}}{E_{b}}\right)^{-1}
$$

and an equivalent curvature radius $\rho_{\mathrm{eq}}=\left(1 / \rho_{\mathrm{eq}_{x}}+1 / \rho_{\mathrm{eq}_{y}}\right)^{-1}$, with

$$
\begin{gathered}
\frac{1}{\rho_{\mathrm{eq}_{x}}}=\frac{1}{r_{a_{x}}}+\frac{1}{r_{b_{x}}}, \\
\frac{1}{\rho_{\mathrm{eq}_{y}}}=\frac{1}{r_{a_{y}}}-\frac{1}{r_{b_{y}}},
\end{gathered}
$$

where the subscript a refers to the roller and $b$ to the disk, and $r_{a_{x}}=\left(r_{0}+e\right) \tan \alpha / 2=r_{2} / \cos (\alpha / 2), r_{b_{x}}=r_{11}, r_{a_{y}}=r_{22}$ and $r_{b_{y}}=r_{0}$ (in the case of half- and full-toroidal $r_{a_{x}}=r_{0}$ ). The equivalent radius of curvature is then defined as

$$
\frac{1}{\rho_{\mathrm{eq}}}=\frac{1}{\rho_{\mathrm{eq}_{x}}}+\frac{1}{\rho_{\mathrm{eq}_{y}}}
$$

In the case of roller-roller conical contact, we define $R_{a_{x}}=$ $R_{b_{x}}=r_{C} / \cos (\alpha / 2)$, being $r_{C}$ the mean rolling radius of the conical part of the rollers, whereas $R_{a_{y}}=R_{b_{y}}=\infty$. We can, then, define the equivalent radius of curvature $R_{\mathrm{eq}_{x}}$ as

$$
\frac{1}{R_{\mathrm{eq}_{x}}}=\frac{1}{R_{\mathrm{a}_{x}}}+\frac{1}{R_{b_{x}}} .
$$


The results of elasto-hydrodynamic theory are employed to describe the lubrication regime on the contact area. In particular, we have considered the viscosity-pressure effect with the Roeland's formula:

$$
\frac{\eta}{\eta_{0}}=\left(\frac{\eta_{\infty}}{\eta_{0}}\right)^{1-\left(1+p / c_{p}\right)^{z_{1}}} .
$$

Because of the very high shear rates, the lubricant shows a nonlinear relation between the shear stresses $\tau_{i j}$ and the shear strain rates $\dot{\varepsilon}_{i j}$, according to the common rule used in the plasticity theory to split the shear strain along the different directions. We write

$$
\frac{\partial v_{i}}{\partial x_{j}}+\frac{\partial v_{j}}{\partial x_{i}}=\frac{\tau_{i j}}{\tau_{e}} \Gamma\left(\tau_{e}\right),
$$

where the function $\Gamma\left(\tau_{e}\right)$ is representative of the nonlinear behavior of the traction oil and

$$
\tau_{e}=\frac{\sqrt{2}}{2}\left(\tau_{i j} \tau_{i j}\right)^{1 / 2}
$$

is the equivalent stress. By following the rheological model proposed by Bair and Winer, the function $\Gamma\left(\tau_{\mathrm{e}}\right)$ takes the form

$$
\Gamma\left(\tau_{e}\right)=\frac{\tau_{L}}{\eta} \ln \left(\frac{1}{1-\tau_{e} / \tau_{L}}\right),
$$

where $\tau_{L}=\tau_{L 0}+a p$ is the limiting shear stress. The estimation of the shear strain is carried out assuming that for hard-EHL conditions, the pressure distribution is close to a Hertzian distribution (except for a peak close to the trailing edge of the contact) and considering that most part of the contact is characterized by an almost constant thickness $h$ of the lubricant film, which, in turn, is calculated on the basis of EHL formulas. Therefore, we can write

$$
\begin{aligned}
& \frac{\partial v_{x}}{\partial z}+\frac{\partial v_{z}}{\partial x} \approx \frac{v_{x}}{h}, \\
& \frac{\partial v_{y}}{\partial z}+\frac{\partial v_{z}}{\partial y} \approx \frac{v_{y}}{h} .
\end{aligned}
$$

Recalling that the local spin-sliding motion is completely determined as a function of the creep, slip, and spin coefficient, one is able to calculate the quantities $\mu_{\text {in }}, \mu_{\text {out }}, \chi_{\text {in }}$, $\chi_{\text {out }}$, and the roller-roller traction coefficient $\mu_{\mathrm{C}}$ by simply integrating the tangential stress over the contact area. Once these quantities are obtained, the efficiency and overall traction performance of the variator are promptly derived (see also $[13,14])$.

\section{Simulation of KERS Behavior}

In this section, we employ the mechanical model of the three toroidal traction drives to investigate the overall performance of a mechanical hybrid powertrain. Among all the possible configurations of mechanical hybrid powertrains [7], a flywheel KERS connected into the vehicle propshaft

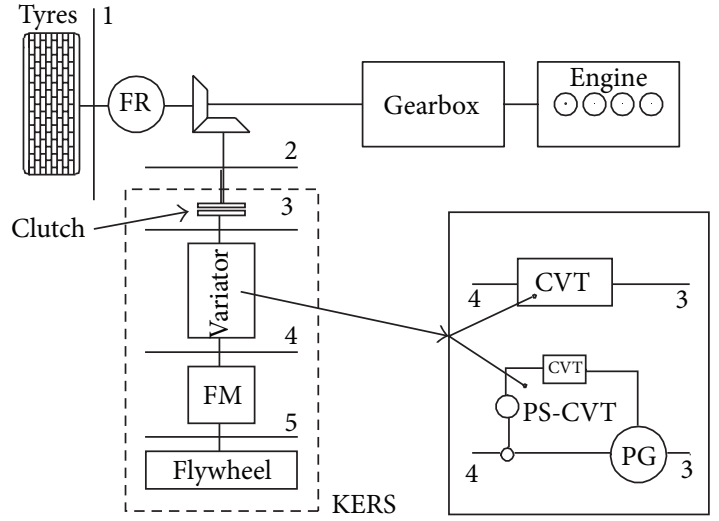

FIGURE 3: A schematic picture of the driveline of the mechanical hybrid vehicle. Sections of the driveline are tagged with numbers. FR is the final drive of the vehicle driveline and FM is the final multiplier. The KERS is plugged in the vehicle propshaft through a friction clutch. A second clutch (not shown in the figure) can disconnect the FM from the variable drive when the flywheel is in idle rotation.

through a friction clutch (see Figure 3) is considered. This configuration gives larger fuel economy improvements than others when it works in synergy with stop and restart system [7]. The simulation has been performed via inverse dynamic simulator of the vehicle powertrain. The driving cycle is given as a velocity pattern, and the driveline parameters are calculated backwards with kinetic, kinematic, and efficiency models. In this paper the focus is on the energy recovery potential of the KERS, and the gearbox and the engine are not included in the model. Further investigation is needed to get the fuel economy and $\mathrm{CO}_{2}$ emissions reduction potential of the KERS. The driveline with KERS, considered in this study, is made of wheels, differential and final drive (FR), KERS driving clutch, toroidal CVT, final step-up drive, and highspeed rotating flywheel. We define two KERS performance indexes: the KERS boost and the round-trip efficiency. In Section 2 of the driveline (see Figure 3), the torque which is necessary to drive the vehicle following the driving schedule is called $T_{2}^{\text {nec }}$, which can be positive or negative. The quantity $T_{2}^{\prime \text { nec }}$ is defined, which is equal to $T_{2}^{\text {nec }}$ only when $T_{2}^{\text {nec }} \geq 0$, zero otherwise. The torque that is actually given by the KERS in Section 2 is $T_{2}$, and it can be positive (reuse mode) or negative (recovery mode). Moreover, we define: $T_{2}^{\prime}$ which is equal to $T_{2}$ if $T_{2} \geq 0$, zero otherwise; $T_{2}^{\prime \prime}$ which is equal to $T_{2}$ if $T_{2}<0$, and zero otherwise. The KERS boost is defined as

$$
\text { KERS boost }=\frac{\int_{0}^{t_{\text {cycle }}} T_{2}^{\prime} \omega_{2} d t}{\int_{0}^{t_{\text {cycle }}} T_{2}^{\prime \text { nec }} \omega_{2} d t},
$$

where $t_{\text {cycle }}$ is the time duration of one driving cycle and $\omega_{2}$ is the angular velocity of shaft 2 . The KERS boost is the energy given by the KERS to the vehicle propshaft per cycle, divided by the energy that is needed to follow the driving schedule per cycle and calculated in Section 2 of the driveline. 
TABLE 1: Fluid properties at $T=99^{\circ} \mathrm{C}$.

Absolute viscosity at the atmospheric pressure

Viscosity-pressure index

Pressure-viscosity coefficient

Limiting shear stress at atmospheric pressure

Limiting shear stress constant

Pole pressure constant of Roelands viscosity model

Pole viscosity of Roelands viscosity model

$$
\begin{gathered}
\eta_{0}=3.25 \times 10^{-3} \mathrm{~Pa} \mathrm{~s} \\
Z_{1}=0.85 \\
\zeta=1.71 \times 10^{-8} \mathrm{~Pa}^{-1} \\
\tau_{L 0}=0.02 \times 10^{9} \mathrm{~Pa} \\
a=0.085 \\
c_{p}=1.96 \times 10^{8} \mathrm{~Pa} \\
\eta_{\infty}=6.31 \times 10^{-5} \mathrm{~Pa} \mathrm{~s}
\end{gathered}
$$

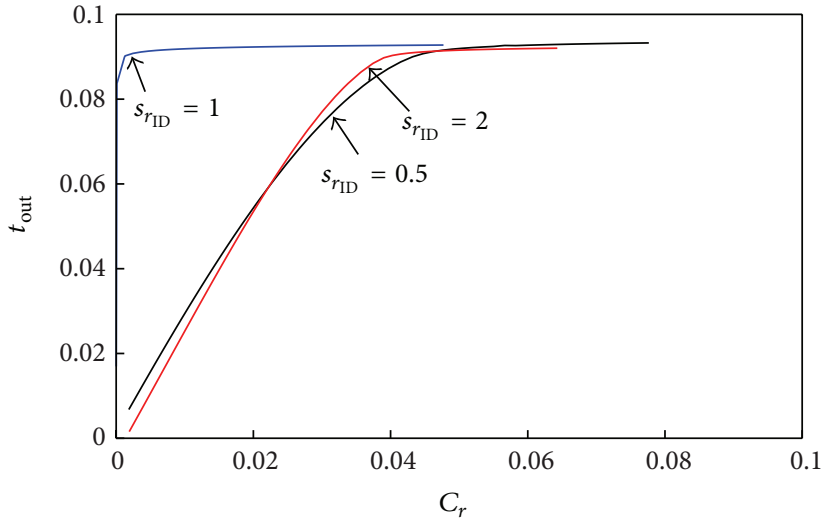

(a)

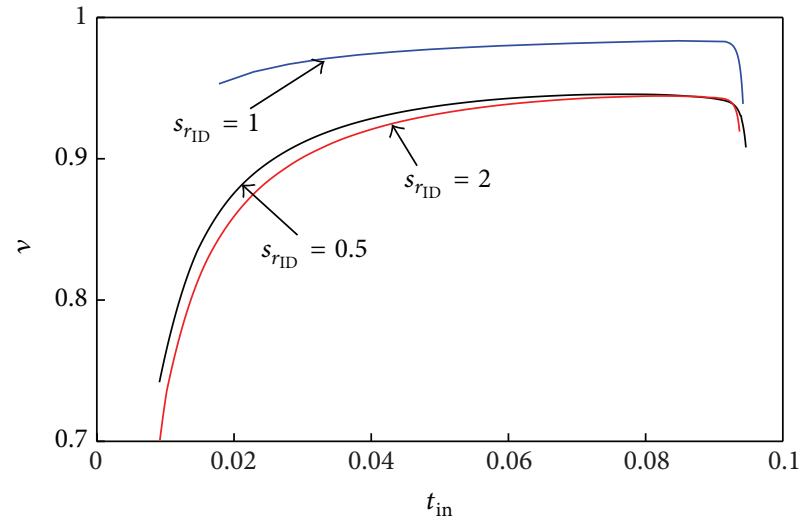

(b)

FIgURE 4: The DFTV variator performance. The dimensionless output torque $t_{\text {out }}$ as a function of the overall creep coefficient $\mathrm{C}_{r}$ (a) and the overall mechanical efficiency $v$ as a function of the input torque coefficient $t_{\text {in }}$ (b) for the three geometric ratios $\left(s_{r_{\mathrm{ID}}}=0.5-\right.$ black curve, $s_{r_{\mathrm{ID}}}=1-$ blue curve, $s_{r_{\mathrm{ID}}}=2-$ red curve). Calculations are shown for $F_{N}=20 \mathrm{kN}$.

The round trip efficiency is calculated as:

$$
\text { Round Trip Efficiency }=\frac{\int_{0}^{t_{\text {cycle }}} T_{2}^{\prime} \omega_{2} d t}{-\int_{0}^{t_{\text {cycle }}} T_{2}^{\prime \prime} \omega_{2} d t},
$$

The round trip efficiency is the energy actually given by the KERS into the vehicle propshaft per cycle divided by the energy actually given by the propshaft to the KERS per cycle.

\section{Results}

In this section, we first present the main traction and efficiency performance of the three investigated toroidal traction drives, and afterwards we focus on the energy recovery efficiency of a KERS geometry based on these kind of transmissions. The fluid properties are reported in Table 1 and the geometrical data of the three variators in Table 2.

5.1. Traction Behavior of the DFTV. Figure 4 shows the performance in terms of traction and efficiency of the double toroidal traction drive (DFTV). Calculations have been carried out at constant values of the primary speed $\omega_{1}=2000 \mathrm{RPM}$ and normal contact load $F_{N}=20 \mathrm{kN}$. Interestingly, since the DFTV variator presents negligible values of spin losses at unit ratio (blue curve in Figure 4(a)), in this case the traction curve increases very fast as the creep is increased from zero. However, at different speed ratios
TABle 2: The geometric data of the three toroid geometries.

\begin{tabular}{lccc}
\hline & SHTV & SFTV & DFTV \\
\hline Cavity radius $r_{0}$ & $40 \mathrm{~mm}$ & $40 \mathrm{~mm}$ & $40 \mathrm{~mm}$ \\
Roller radius $r_{2}$ & $34.6 \mathrm{~mm}$ & $40 \mathrm{~mm}$ & $18.7 \mathrm{~mm}$ \\
Conformity ratio CR $=r_{22} / r_{0}$ & 0.8 & 0.5 & 0.8 \\
Half-cone angle $\theta$ & $\pi / 3$ & $\pi / 2$ & $\pi / 2$ \\
Incidence cone-angle $\alpha$ & & & $43 \mathrm{deg}$ \\
Aspect ratio $k=e / r_{0}$ & 0.625 & 0.25 & 0.286 \\
$N^{\circ}$ of cavities $m$ & 2 & 2 & 2 \\
$N^{\circ}$ of rollers set $n$ & 3 & 3 & 3 \\
$N^{\circ}$ of rollers per set $n_{r}$ & 1 & 1 & 2 \\
\hline
\end{tabular}

$s_{r_{\mathrm{ID}}}=0.5$ (red curve in Figure $4(\mathrm{a})$ ), and $s_{r_{\mathrm{ID}}}=2$ (black curve in Figure 4(a)), the presence of spin losses determines a strong decrease of traction versus creep slope. The linear behavior is observed until a creep value $C_{r}=0.02$ is reached, where the traction curve presents a typical knee that corresponds to the maximum achievable value of output dimensionless torque $\left(t_{\text {out }}\right)_{\max } \approx 0.095$. In Figure 4(b) we show the variator mechanical efficiency $v$ as a function of the dimensionless input torque $t_{\text {in }}$. It is worth noticing that at $s_{r_{\mathrm{ID}}}=1$, the efficiency takes very high values, equal to about 0.98 on most part of the input torque range. However, as $t_{\text {in }}$ (and therefore $t_{\text {out }}$ ) approaches its limiting value the efficiency rapidly drops because of the fast increase of the 


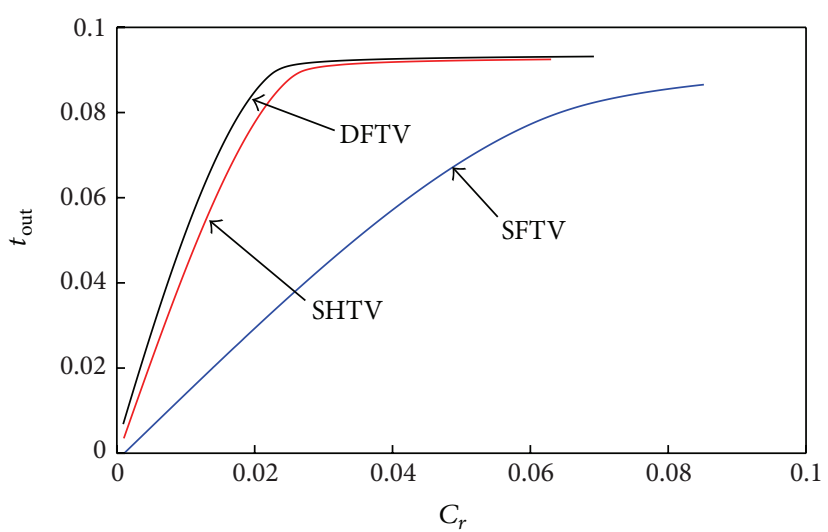

(a)

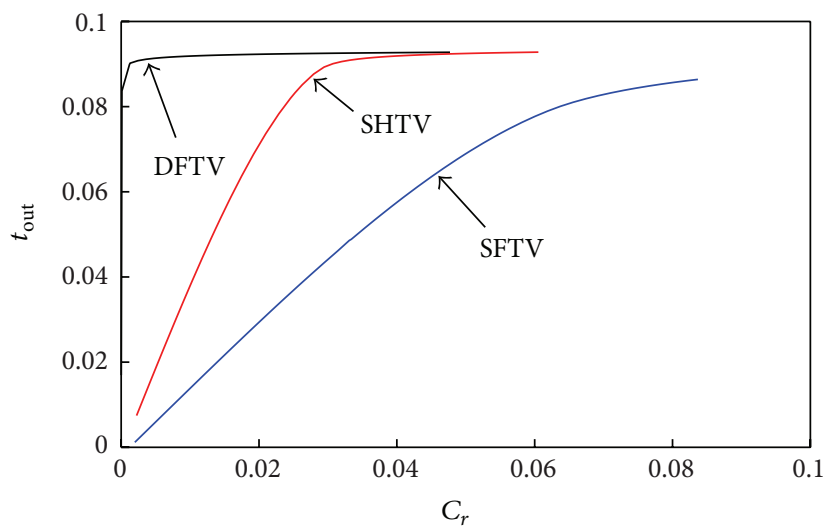

(c)

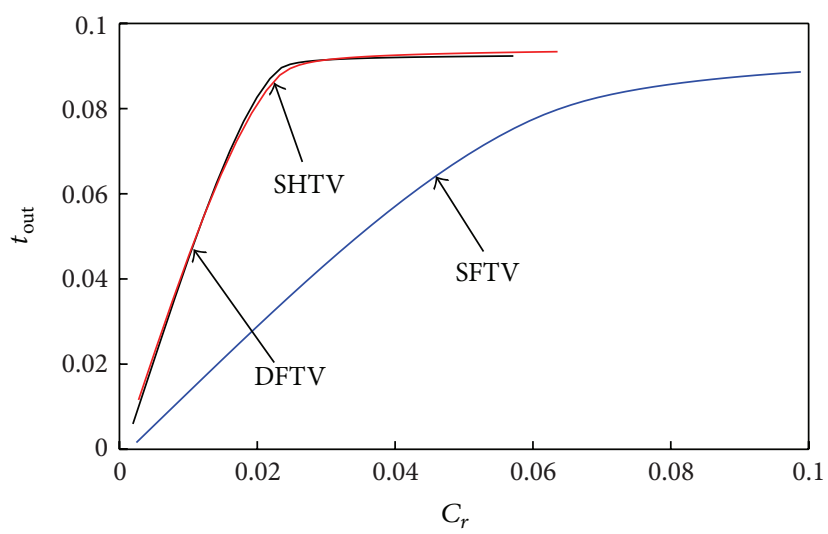

(e)

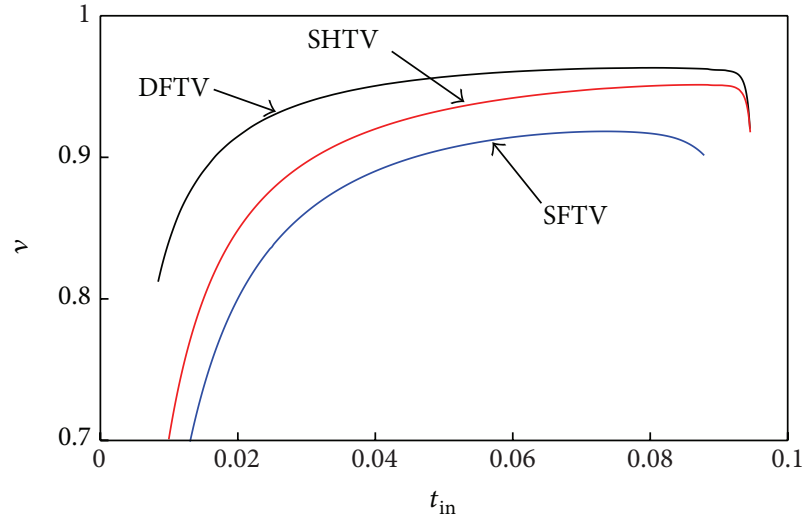

(b)

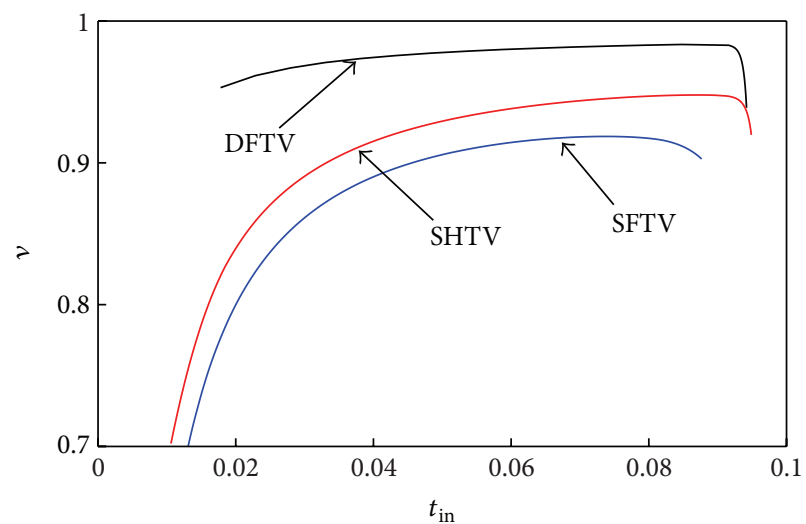

(d)

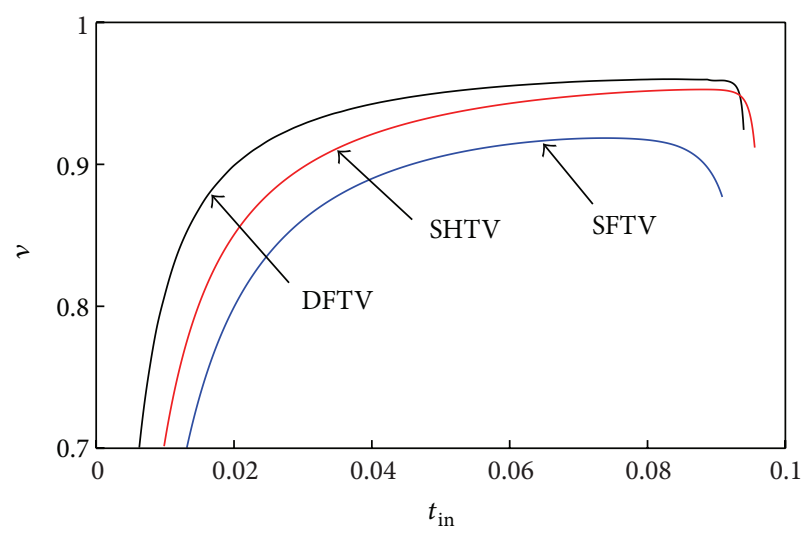

(f)

FIGURE 5: The effective output torque $t_{\text {out }}$ as a function of the overall creep coefficient $C_{r}$ and the overall efficiency $v$ as a function of the effective input torque $\mathrm{s}_{r_{\mathrm{ID}}}=1$, for DFTV, SHTV, and SFTV. Calculations are presented for a normal load $F_{N}=20 \mathrm{kN}$.

creep coefficient. At the extreme ratios: $s_{r_{10}}=0.5$ (red curve), $s_{r_{\mathrm{ID}}}=2$ (black curve) in Figure 4(b), the trend of the $v$ versus $t_{\text {in }}$ resembles the behavior observed for $s_{r_{\mathrm{ID}}}=1$, but the efficiency values are considerably smaller over the whole torque range, with a maximum value close to $0.94-0.95$.

5.2. Comparison of DFTV, SFTV, and SHTV. Figure 5 compares quantitatively the different toroidal variators in terms of traction capabilities and efficiency. Calculations have been carried out at given constant values of the primary speed $\omega_{1}=2000 \mathrm{RPM}$ and of the normal load $F_{N}=20 \mathrm{kN}$. The dimensionless output torque $t_{\text {out }}$ is plotted against the overall creep coefficient $C_{r}$, whereas the efficiency $v$ is represented as a function of the input dimensionless torque $t_{\text {in }}$. In all cases, we observe, as expected, an almost linear increase of $t_{\text {out }}$ as $C_{r}$ is increased from zero. However, as the creep increases, the curve starts to deviate from the linear trend and reaches a saturation value in correspondence of the 
maximum transmissible torque. Interestingly, the slope of the linear part of the three traction curves is different for the three different variators and also changes as the geometric speed ratio $s_{r_{\mathrm{ID}}}$ is changed. In all cases, the SFTV shows a significantly worse behavior if compared to the other two typologies. More interesting is to compare the SHTV and the DFTV. At ratios $s_{r_{\mathrm{ID}}}=0.7$ and $s_{r_{\mathrm{ID}}}=1.5$, the two variators show almost the traction capabilities however the DFTV performs significantly better than the SHTV in terms of mechanical efficiency. The DFTV strongly outperforms both in terms of traction and mechanical efficiency the SHTV at geometric speed ratio $s_{r_{\mathrm{ID}}}=1$, as a consequence of the very limited amount of spin motion and spin momentum.

5.3. KERS Performance. The simulations of KERS performance have been carried out with the data of a typical city car (more details are in [15]). The main features of the KERS device follow. We have considered a CVT KERS mass equal to about $25 \mathrm{~kg}$. The simulations have been performed considering a flywheel with the following characteristics: flywheel inertia $J_{f}=0.0562 \mathrm{kgm}^{2}$, flywheel minimum velocity $\omega_{f_{\min }}=12 \mathrm{kRPM}$, and flywheel maximum velocity $\omega_{f_{\max }}=24 \mathrm{kRPM}$ (upper and lower bounds have been chosen according to [17]). The mechanical hybrid under analysis can be exploited for urban driving applications. The maximum energy which can be stored in the flywheel is about $178 \mathrm{~kJ}$ that corresponds to the kinetic energy of the vehicle at $60 \mathrm{kph}$. The simulations have been performed following the urban FTP-75 driving schedule. The driving schedule has been considered as a periodic function which must be followed by the vehicle. The state of charge of the flywheel is the same at the beginning and at the end of the cycle [4]. In order to compare the performance which can be achieved with different variators, for any given toroidal traction drive under investigation, the speed ratio $\tau_{\mathrm{FM}}$ of the final multiplier has been optimized and this optimal value has been used to carry out the simulations. The mechanical efficiencies of DFTV, SHTV, and SFTV have been calculated by means of the analytical models presented in previous sections. We assume that the clamping system allows to control the clamping force in order to optimize the efficiency of the toroidal traction drive for any given speed ratio and the input torque. In order to perform simulations under these working conditions, the efficiency has been calculated with the optimal value of the normalized input torque $t_{\text {in }}$ with different speed ratios, and the results are shown in Figure 6. The ratio spread of the three variators is equal to 4 , with the speed ratio ranging from $s_{r_{\mathrm{IF}}}=0.5$ to $s_{r_{\mathrm{IF}}}=2.0$. It is shown that the efficiency of the SFTV is smaller than the efficiency of the DFTV and the SHTV over the entire range of speed ratios. The DFTV outperforms the SHTV only in the intermediate range of speed ratio values. The results of our simulations are shown in Figure 7. The KERS boost (see Figure 7(a)) in the FTP-75 driving schedule is equal to $20.4 \%$ with DFTV and $20.2 \%$ with SHTV (the difference is almost negligible), whereas it is equal to $18.4 \%$ in the case of SFTV. Because SFTV is the one which is currently used in mechanical hybrid systems, our results show that a correct choice of the variator

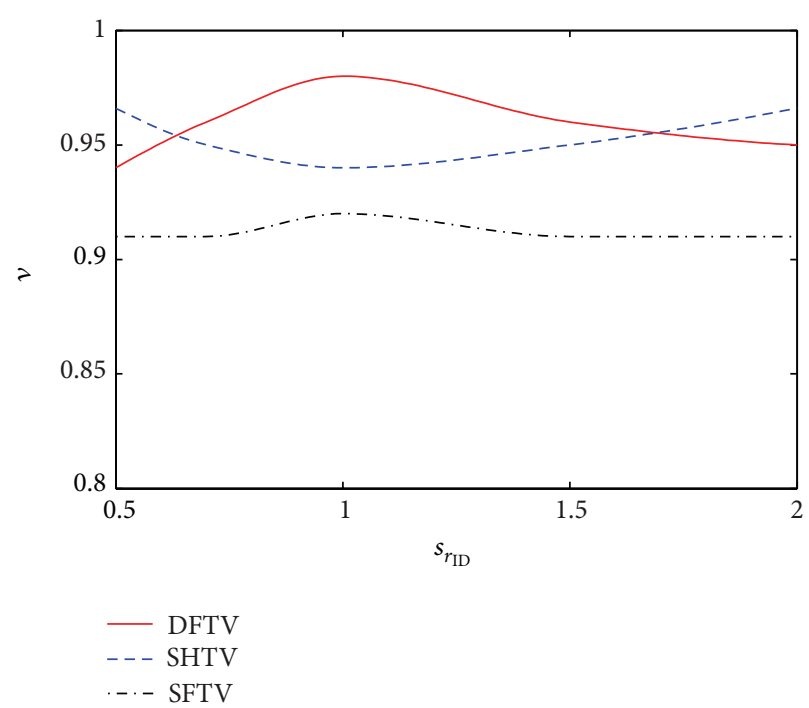

FIgURE 6: The optimal efficiency values of three toroidal CVTs as a function of the speed ratio $\mathrm{s}_{r_{\mathrm{ID}}}$.

may lead to an improvement of the KERS boost of about $10 \%$. Similar results are shown in FTP driving schedule. In this case, the KERS boost is about $10.2 \%$ with SFTV, $11.3 \%$ with SHTV, and $11.5 \%$ with DFTV. As expected, the best performance are obtained in the urban drive. Figure 7(b) shows the overall round-trip efficiency of the KERS in FTP75 and FTP driving schedules and a comparison of the results achieved with SFTV, SHTV and DFTV. The round-trip efficiency is not much affected by the driving style, whereas the SFTV efficiency is about 7\% smaller in comparison to the DFTV and the SHTV. In our analysis, we have considered a ratio spread close to 4 for all the toroidal traction drives. However, we notice that a variable drive with a larger ratio spread may perform even better provided that the efficiency remains sufficiently high [15]. However, the twofold objective of designing a variator which possesses both large ratio spread and high mechanical efficiency over the entire speed ratio range is a quite challenging task. Different variators may provide different room for further improvement, and, from this point of view, further investigations should deal with the optimization of the design of the DFTV and SHTV for application to KERS.

\section{Conclusions}

We have analyzed the efficiency and traction performance of three toroidal traction drives: the SFTV, the SHTV, and the recently patented DFTV. The latter has been designed in such a way to combine the advantages of the other two existing toroidal geometries and shows enhanced efficiency and traction performance. The DFTV variator consists of a set of counter-rotating conical rollers which are placed into a toroidal cavity; the geometric characteristics of the power rollers allow to reduce the spin losses in a wide range of speed ratios and to get rid of the thrust ball-bearing, which contributes to the torque losses of the half-toroidal type 


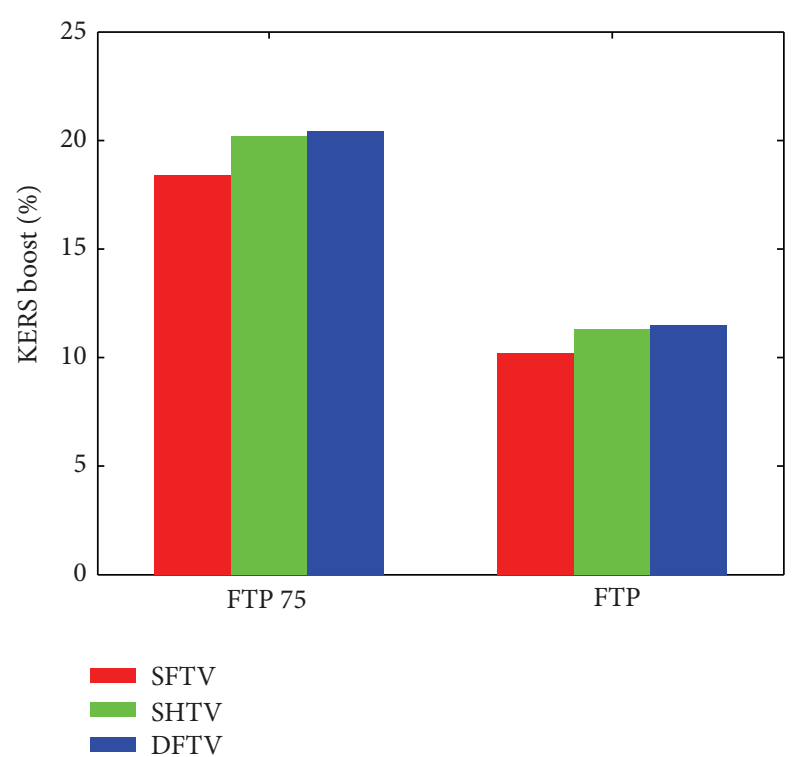

(a)

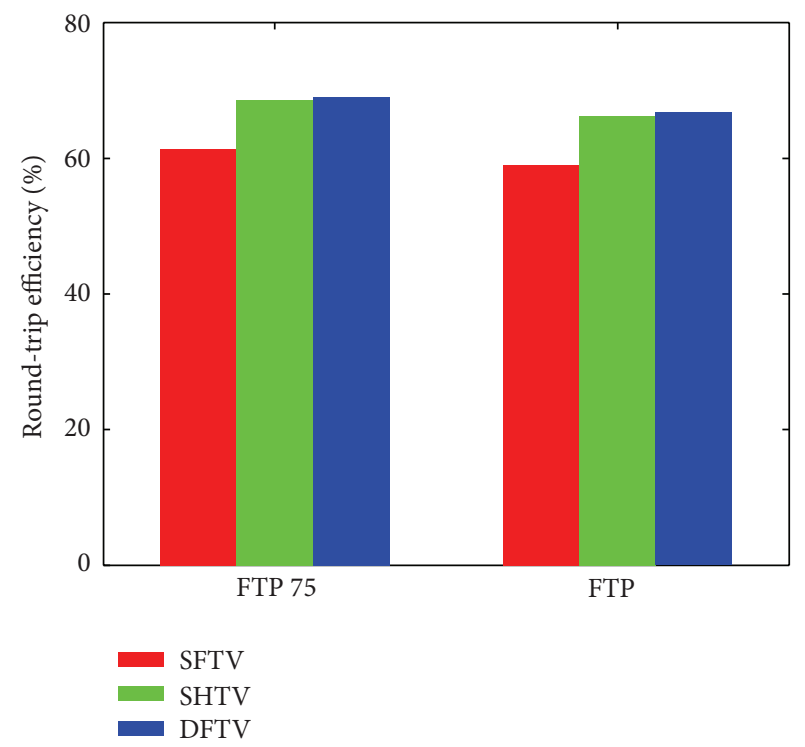

(b)

FIGURE 7: The KERS boost (a) and the KERS round trip efficiency (b) calculated in FTP 75 (urban) and FTP (mixed) driving schedules.

geometry. In order to evaluate the performance of the toroidal traction drives, we have developed a fully flooded isothermal contact model, based on the results of EHL lubrication theory. Our calculations have shown the effectiveness of the DFTV geometric characteristics in terms of reduction of spin losses and improvement of the overall efficiency. The very high mechanical efficiency and traction performance of the DFTV have then been exploited to investigate the performance of a mechanical Kinetic Energy Recovery System (KERS). The energy boost capabilities and the overall round trip efficiency have been calculated for DFTV, SHTV, and SFTV, and a comparison has been discussed. The results have shown that the choice of DFTV and SHTV leads to a very significant increase of the KERS boost capability in urban drive, which is about $10 \%$ larger than the result achieved with SFTV.

\section{References}

[1] G. Mantriota, "Fuel consumption of a vehicle with power split CVT system," International Journal of Vehicle Design, vol. 37, no. 4, pp. 327-342, 2005.

[2] C. Brace, M. Deacon, N. D. Vaughan, R. W. Horrocks, and C. R. Burrows, "The compromise in reducing exhaust emissions and fuel consumption from a diesel CVT powertrain over typical usage cycles," in Proceedings of the Inernational Congress on Continuously Variable Power Transmission (CVT '99), pp. 2733, Eindhoven, The Netherlands, September 1999.

[3] G. Carbone, L. Mangialardi, and G. Mantriota, "Fuel consumption of a mid class vehicle with infinitely variable transmission," SAE International Journal of Engines, vol. 110, no. 3, pp. 24742483, 2002.

[4] D. Cross and C. Brockbank, "Mechanical hybrid system comprising a flywheel and CVT for motorsport and mainstream automotive applications," SAE Technical Paper 2009-01-1312.
[5] C. Brockbank, "Development of full-toroidal traction drives in flywheel based mechanical hybrids," in Proceedings of the CVT Hybrid International Conference (CVT '10), pp. 163-169, Maastricht, The Netherlands, November 2010.

[6] A. Barr and A. Veshnagh, "Fuel economy and performance comparison of alternative mechanical hybrid powertrain configurations," SAE Technical Paper 2208-01-0083.

[7] C. Brockbank and W. Body, "Flywheel based mechanical hybrid system; simulation of the fuel consumption benefits of various transmission arrangements and control strategies," in Proceedings of the ASME International Design Engineering Technical Conferences \& Computers and Information in Engineering Conference (IDETC/CIE '10), Montreal, Canada, August 2010.

[8] G. Carbone, L. De Novellis, G. Commissaris, and M. Steinbuch, "An enhanced CMM model for the accurate prediction of steady-state performance of CVT chain drives," Journal of Mechanical Design, Transactions of the ASME, vol. 132, no. 2, Article ID 021005, 8 pages, 2010.

[9] L. De Novellis and G. Carbone, "Experimental investigation of chain link forces in continuously variable transmissions," Journal of Mechanical Design, Transactions of the ASME, vol.132, no. 12, Article ID 121004, 2010.

[10] G. Carbone, L. Mangialardi, and G. Mantriota, "Influence of clearance between plates in metal pushing V-belt dynamics," Journal of Mechanical Design, Transactions of the ASME, vol.124, no. 3, pp. 543-557, 2002.

[11] T. Imanishi and H. Machida, "Development of powertoros unit half-toroidal CVT (2)-comparison between half-toroidal and full-toroidal CVTs," Motion \& Control, no. 10, 2001.

[12] M. J. Durack, "Full toroidal traction drive, world intellectual property organization," International Publication WO 2011/041851 A1, http://worldwide.espacenet.com/.

[13] G. Carbone, L. Mangialardi, and G. Mantriota, "A comparison of the performances of full and half toroidal traction drives," Mechanism and Machine Theory, vol. 39, no. 9, pp. 921-942, 2004. 
[14] L. De Novellis, G. Carbone, and L. Mangialardi, "Traction and efficiency performance of the Double roller Full Toroidal Variator: a comparison with Half- and Full- Toroidal drives," ASME Journal of Mechanical Design, vol. 134, no. 7, Article ID 071005, 14 pages, 2012.

[15] F. Bottiglione and G. Mantriota, "Effect of the ratio spread of CVU in automotive Kinetic Energy Recovery Systems," ASME Journal of Mechanical Design, In Press.

[16] L. Mangialardi and G. Mantriota, "Power flows and efficiency in infinitely variable transmissions," Mechanism and Machine Theory, vol. 34, no. 7, pp. 973-994, 1999.

[17] C. J. Greenwood, "An energy recovery system for a vehicle driveline," World Intellectual Property Organization, International Publication WO2009/141646 A1, http://worldwide.espacenet .com/.

[18] F. Bottiglione and G. Mantriota, "Reversibility of power-split transmissions," Journal of Mechanical Design, vol. 133, no. 8, Article ID 084503, 5 pages, 2011.

[19] SKF General Catalogue 2003.

[20] B. J. Hamrock, Fundamentals of Fluid Film Lubrication, Series in Mechanical Engineering, Book, McGraw-Hill, 1994. 

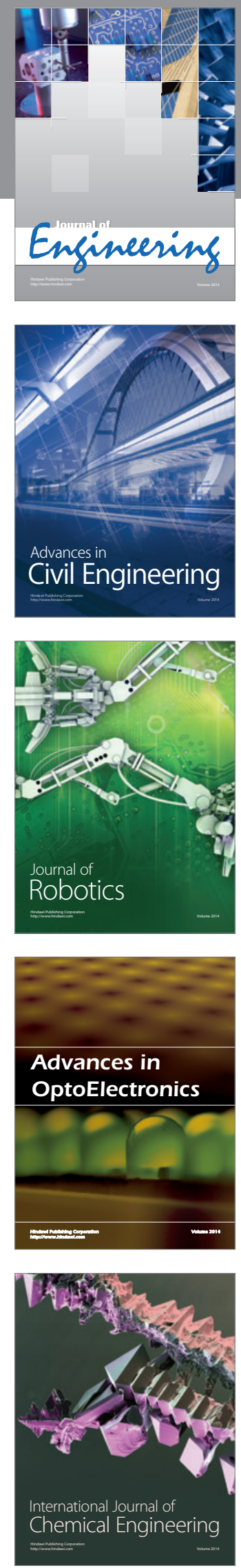

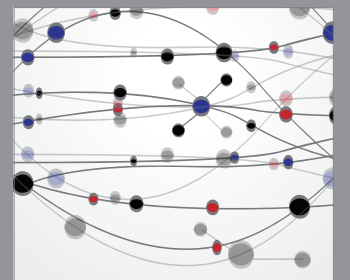

The Scientific World Journal
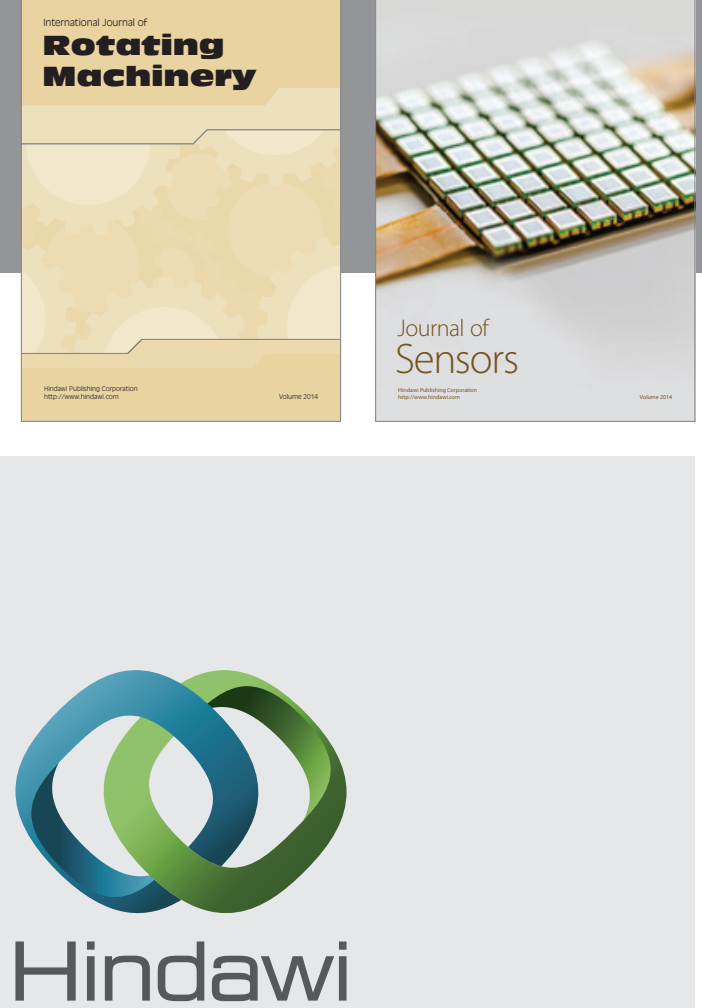

Submit your manuscripts at http://www.hindawi.com
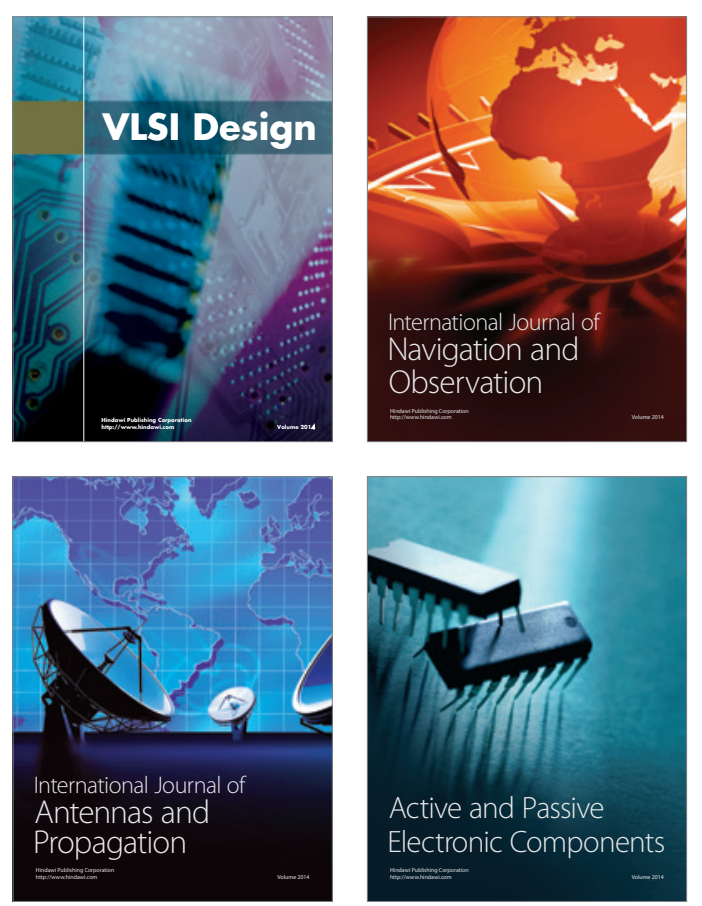
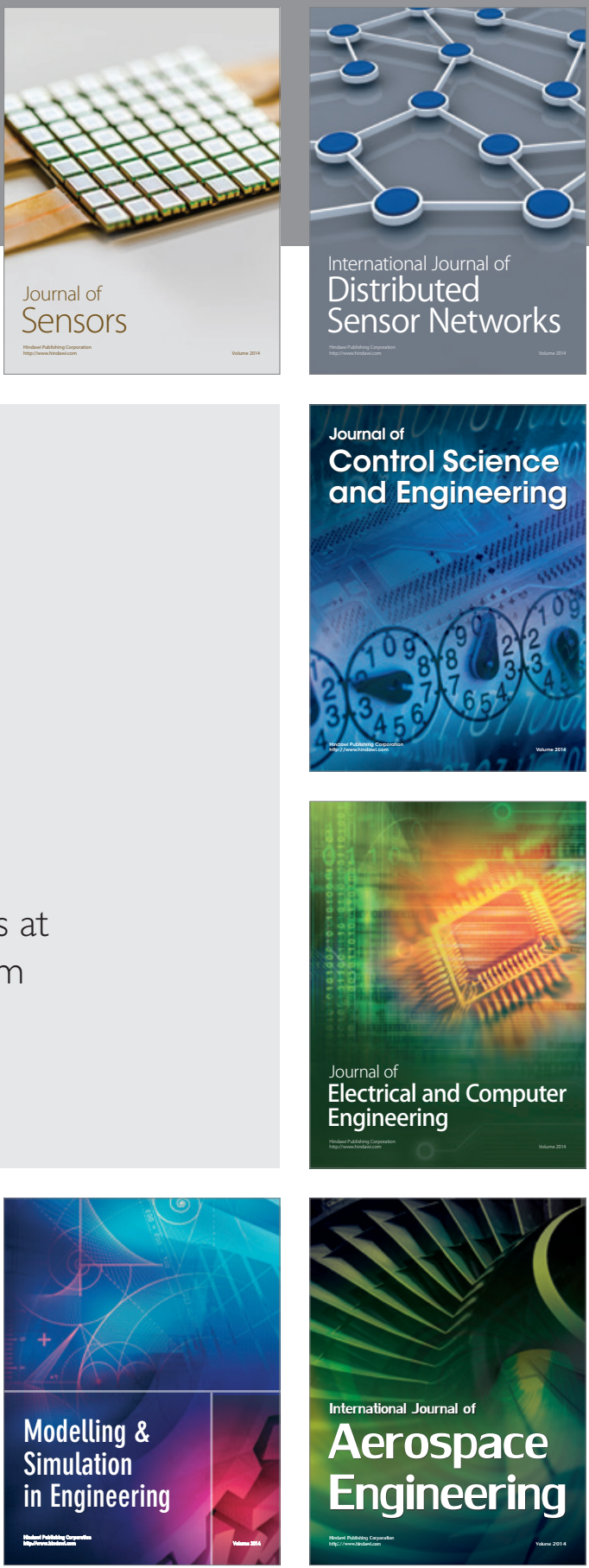

Journal of

Control Science

and Engineering
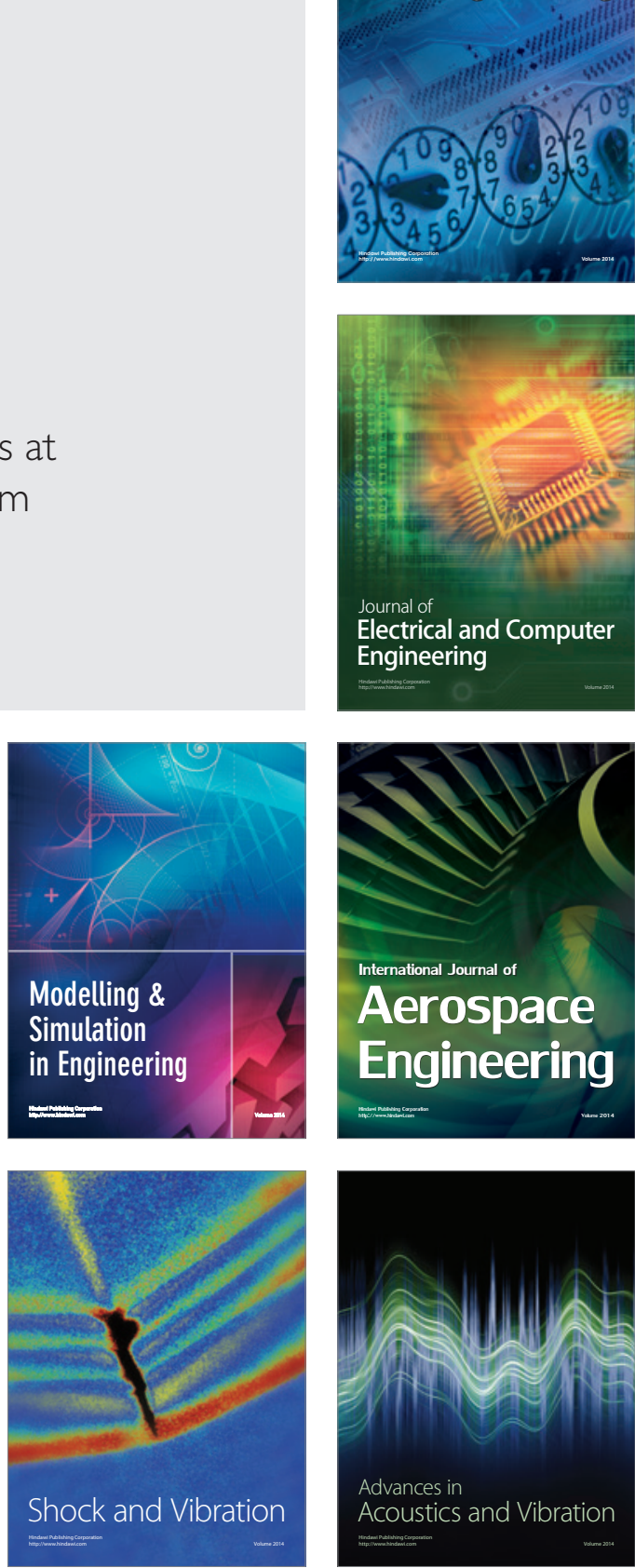